\title{
Assessing the role of quantum effects in two-dimensional heterophase MoTe2 field effect transistors
}

Jelver, Line; Hansen, Ole; Jacobsen, Karsten Wedel

Published in:

Physical Review B

Link to article, DOI:

10.1103/PhysRevB.104.045313

Publication date:

2021

Document Version

Publisher's PDF, also known as Version of record

Link back to DTU Orbit

Citation (APA):

Jelver, L., Hansen, O., \& Jacobsen, K. W. (2021). Assessing the role of quantum effects in two-dimensional heterophase MoTe field effect transistors. Physical Review B, 104(4), [045313].

https://doi.org/10.1 f03/PhysRevB.104.045313

\section{General rights}

Copyright and moral rights for the publications made accessible in the public portal are retained by the authors and/or other copyright owners and it is a condition of accessing publications that users recognise and abide by the legal requirements associated with these rights.

- Users may download and print one copy of any publication from the public portal for the purpose of private study or research.

- You may not further distribute the material or use it for any profit-making activity or commercial gain

- You may freely distribute the URL identifying the publication in the public portal 


\title{
Assessing the role of quantum effects in two-dimensional heterophase $\mathrm{MoTe}_{2}$ field effect transistors
}

\author{
Line Jelver $\odot^{*}$ \\ CAMD, Dept. of Physics, Technical University of Denmark, Bldg. 309, DK-2800 Kongens Lyngby, Denmark; \\ Synopsys QuantumATK, Fruebjergvej 3, PostBox 4, DK-2100 Copenhagen, Denmark; \\ and Center for Nano Optics, University of Southern Denmark, DK-5230 Odense M, Denmark \\ Ole Hansen 10 \\ DTU Nanolab, Technical University of Denmark, Bldg. 347, DK-2800 Kongens Lyngby, Denmark \\ Karsten Wedel Jacobsen $\odot$ \\ CAMD, Dept. of Physics, Technical University of Denmark, Bldg. 309, DK-2800 Kongens Lyngby, Denmark
}

(Received 18 May 2021; revised 5 July 2021; accepted 7 July 2021; published 27 July 2021)

\begin{abstract}
The two-dimensional (2D) transition metal dichalcogenides (TMDs) have been proposed as candidates for the channel material in future field effect transistor designs. The heterophase design, which utilizes the metallic $\mathrm{T}$ or $\mathrm{T}^{\prime}$ phase of the TMD as contacts to the semiconducting H-phase channel, has shown promising results in terms of bringing down the contact resistance of the device. In this work, we use ab-initio calculations to demonstrate how atomic-scale and quantum effects influence the ballistic transport properties in such heterophase transistors with channel lengths up to $20 \mathrm{~nm}$. We investigate how the charge transfer depends on the carrier density both in $\mathrm{T}^{\prime}-\mathrm{H} \mathrm{MoTe} \mathrm{M}_{2}$ Schottky contacts and in planar $\mathrm{T}^{\prime}-\mathrm{H}_{-} \mathrm{T}^{\prime} \mathrm{MoTe}_{2}$ transistors. We find that the size of the Schottky barrier and the charge transfer is dominated by the local atomic arrangements at the interface and the doping level. Furthermore, two types of quantum states have a large influence on the charge transport: Interface states and standing waves in the semiconductor due to quantum confinement. We find that the latter can be associated with rises in the current by more than an order of magnitude due to resonant tunneling. Our results demonstrate the quantum mechanical nature of these 2D transistors and highlight several challenges and possible solutions for achieving a competitive performance of such devices.
\end{abstract}

DOI: 10.1103/PhysRevB.104.045313

\section{INTRODUCTION}

Field effect transistors (FETs) have through several decades been continuously optimized and downscaled. Today, quantum effects have become one of the limiting factors in the device performance. Tunneling effects between the source and the drain as well as through the gate stack make it challenging to continue the downscaling. Further development of the energy efficiency and computational power is, therefore, to a larger and larger degree achieved by optimizing the transistor design and materials [1].

The two-dimensional (2D) transition metal dichalcogenides (TMDs) represent a promising group of materials, which could substitute silicon as the semiconducting channel of the device. These 2D semiconductors have the advantage that they, in contrast to silicon, can be synthesized down to the monolayer (ML) limit while retaining a high mobility [2]. This allows for the ultimate downscaling of the transistor channel with a typical ML thickness of only $0.7 \mathrm{~nm}$. However, two large challenges need to be resolved before these materials can become a viable choice for future transistor designs. First, a scalable method for area selective doping of 2D materials is not currently available [3]. This means that the

\footnotetext{
*lije@mci.sdu.dk
}

carrier density in a 2D semiconductor cannot be controlled in a reproducible manner. Second, the interface between the 2D channel and the 3D source and drain contacts is difficult to fabricate. Metal deposition on a 2D material can be very destructive and impurities and defects in the interface result in large contact resistances [4,5]. A promising solution to this is to exploit that the TMDs exist in different phases which exhibit either metallic or semiconducting properties. The group-IV TMDs are generally most stable in the semiconducting $2 \mathrm{H}$ phase but in $\mathrm{MoTe}_{2}$, the metallic $1 \mathrm{~T}^{\prime}$ phase is metastable [6] and can be selectively grown [7-13] or induced from a $2 \mathrm{H}$ sample $[14,15]$. The $\mathrm{MoTe}_{2}$ heterophase transistor design relies on this fact and utilizes the $1 \mathrm{~T}^{\prime}$ phase of the TMD as the source and drain contact. This approach reduces the contact resistance significantly since charge transport across the interface between $1 \mathrm{~T}^{\prime}-\mathrm{MoTe}_{2}$ and a $3 \mathrm{D}$ metal contact seems to be less vulnerable to impurities and defects [8-14]. The ultimate prospect of this design is to combine the ML versions of these bulk phases, the $\mathrm{H}_{\text {and }} \mathrm{T}^{\prime}$ phases, to create a completely $2 \mathrm{D}$ transistor.

$A b$-initio and first-principles-based methods have been extensively used to model 2D material-based devices during recent years [16-32]. In our previous work [31], we have used $a b$-initio calculations to demonstrate how interface resonances at ML $\mathrm{T}^{\prime}-\mathrm{H} \mathrm{MoTe}_{2}$ contacts create peaks in the transmission such that the Schottky barrier heights extracted 
by the thermionic emission model yield significantly different results than the Schottky barrier seen in the density of states (DOS). In this work, we go a step further and investigate the consequences of such quantum effects not only in the Schottky contacts but also in the $\mathrm{T}^{\prime}-\mathrm{H}-\mathrm{T}^{\prime} \mathrm{MoTe}_{2}$ transistors.

The investigations are conducted in two steps. The first step is to examine the Schottky barriers and charge transport in two different $\mathrm{T}^{\prime}-\mathrm{H} \mathrm{MoTe} \mathrm{M}_{2}$ contacts as the carrier density is gradually increased. FETs operate by adjusting an electrostatic field using the gate electrode to draw in or push out carriers in the semiconductor channel. The same effect can, therefore, be created by gradually increasing or decreasing the carrier density in the semiconductor at a single metal-semiconductor interface. Previous ab-initio studies by Saha et al. [20] and Urquiza et al. [22] have investigated two different doping densities of $\mathrm{T}^{\prime}-\mathrm{H} \mathrm{MoS}_{2}$ Schottky contacts and concluded that an increased doping density decreases both the barrier height and the depletion width, and our previous work [31] has demonstrated the same effect in $\mathrm{T}^{\prime}-\mathrm{H} \mathrm{MoTe}_{2}$ contacts. However, to our knowledge, this is the first time the properties of these Schottky contacts have been investigated by gradually changing the carrier density. This gradual change allows for mimicking the effects of an applied gate potential in a FET and the calculations, therefore, provide new fundamental understanding of the electrostatic control over the charge transfer in these $2 \mathrm{D}$ contacts.

The second step is to directly study the charge transport in $\mathrm{MoTe}_{2}$ heterophase FETs. The two contacts investigated in the previous part are combined to form complete 2D FETs with channel lengths of 10,18 , and $20 \mathrm{~nm}$. In the case of the $20-\mathrm{nm}$ channel, the self-consistent transport calculations include 573 atoms, which takes this ab-initio approach to the limit of what is computationally practical. Devices on this length scale have not previously been investigated and help to shed light on the behavior of heterophase FETs in a regime where the channel lengths are comparable to channel lengths used in commercial devices [1].

Our analysis of the Schottky contacts demonstrates that the local atomic configuration at the interface completely dominates the Schottky barrier height, depletion width, and charge transfer. Furthermore, we find that quantum effects play an important part in the charge transfer at the heterophase devices. In both cases of the contacts and the transistors, quantum states bridge the Schottky barrier and contribute significantly to the total current. In the contact, interface states increase the transmission probability and lowers the effective Schottky barrier height. In the transistors, standing waves in the channel increase this effect and results in large current fluctuations in the transfer characteristics, even at a channel length of $20 \mathrm{~nm}$. However, we find that our devices are not competitive with silicon technology either in terms of efficiency or output current. The largest challenges for this heterophase design is that the Schottky barrier at the source, which partly determines the efficiency, is controlled by the local atomic configuration and carrier density in the channel and that the ballistic ON-current is inhibited due to the restriction of momentum conservation along the interface.

The paper is organized as follows. In Sec. II, we explain the details regarding the applied methods and the setup of the Schottky contacts and heterophase transistors. In Sec. III, we demonstrate how the Schottky contacts respond to increasing the carrier density. This part serves as background to understand the results of the complete transistor simulations which are the subject of Sec. IV. After demonstrating the results of the device simulations, we discuss how these calculations compare to previous experiments and calculations on the $\mathrm{MoTe}_{2}$ heterophase transistor and how the performance of these devices could be optimized. This is the subject of Sec. V. Finally, we summarize the main conclusions.

\section{METHOD}

Interfaces between the two phases of $\mathrm{MoTe}_{2}$ are most commonly observed along the zigzag direction of both phases or along the zigzag direction of the $\mathrm{H}$ phase and at an angle of $60^{\circ}$ to the zigzag direction of the T' phase $[8,33,34]$. Here we choose to investigate interfaces along the zigzag direction. In this direction, the unit cells of the $\mathrm{T}^{\prime}$ - and $\mathrm{H}$-phase TMD can be combined in eight different ways while conserving the stoichiometry of the two phases. A stability analysis of these eight interfaces is included in the Supplemental Material Ref. [35]. The c- and d-types are chosen, since these represent some of the most stable interfaces, the c-type in Te-rich conditions and the d-type in intermediate and Mo-rich conditions, meanwhile demonstrating the large variance in interface dipole represented by the different interface geometries.

The c- and d-type interfaces are shown in the middle part of Fig. 1. Both of these interfaces have been found by combining the two unit cells along the zigzag direction and thereby creating a strain of $2.12 \%$ in the $y$ direction of the $\mathrm{T}^{\prime}$ phase. The three unit cells of each phase closest to the interface have been relaxed to a force tolerance of $0.02 \mathrm{eV} / \AA$. The unit cell is doubled in the $y$ direction compared with the conventional unit cells to allow for a stabilizing distortion at the interface. This distortion is clearly visible at the d-type interface.

We use density functional theory $[36,37]$ with the PerdewBurke-Ernzerhof (PBE) exchange-correlation functional [38] and the nonequilibrium Green's function method as implemented in QuantumATK [39]. The wave functions are expanded as linear combinations of atomic orbitals using PseudoDojo pseudopotentials [40] using a cut-off energy of $100 \mathrm{Ha}$. The method is described in detail in our previous work [31] on similar interfaces where we also justify using $\mathrm{PBE}$ to describe these systems. Within this description, the $\mathrm{H}$ phase is a direct band gap semiconductor with a band gap of $1.03 \mathrm{eV}$ and the $\mathrm{T}^{\prime}$ phase is gapless. The computational cell of the Schottky contacts is shown in Fig. 1(a). The total cell size of the central region is $(30.0,0.718,15.0) \mathrm{nm}$ which is sampled by $6 \mathrm{k}$-points in the $y$ direction and a single point in the $z$ direction. The $\mathrm{k}$-grid of the electrodes is $(401,6,1)$ which is necessary to obtain the correct self-energies for the transport calculations. Figure 1(b) shows the computational cell of the 2D planar FETs, which combines a c- and d-type interface as the drain and source contacts, respectively. The symmetry of the $\mathrm{H}$ phase inhibits using the same interface geometry at both source and drain, which is why the device is a combination of the two different geometries. The cell sizes of the central region are $\left(10.0+L_{C}, 0.718,15.0\right) \mathrm{nm}$, where $L_{C}$ is either 10.0, or 18.0, or $20.0 \mathrm{~nm}$ and the k-space grids are equivalent to the Schottky contact calculations. 
(a)

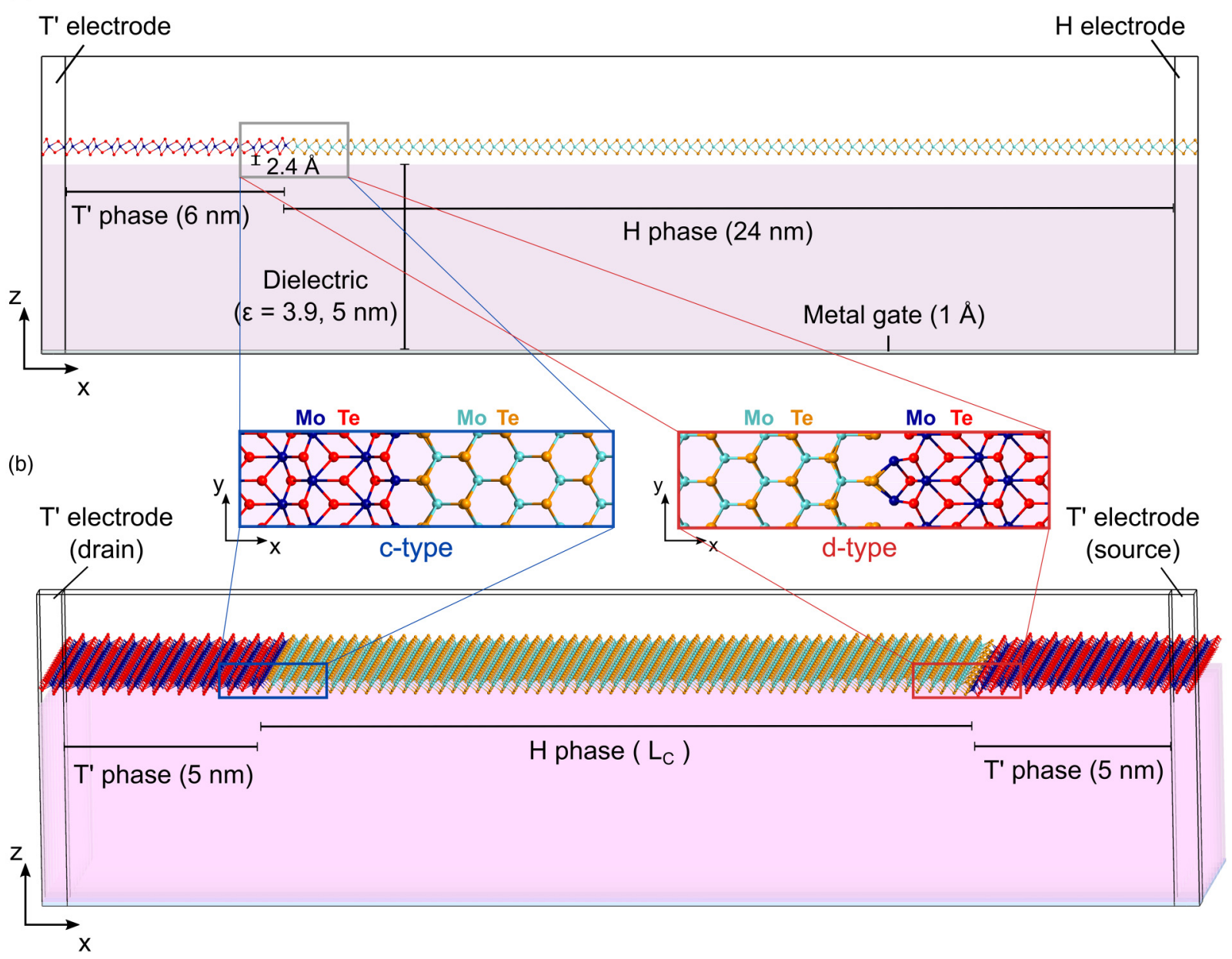

FIG. 1. The $\mathrm{T}^{\prime}-\mathrm{H}$ Schottky contact and $\mathrm{T}^{\prime}-\mathrm{H}-\mathrm{T}^{\prime}$ transistor of ML MoTe 2 on a substrate and back gate. (a) The computational cell of the contacts consisting of a central region and a $\mathrm{T}^{\prime}$ - and $\mathrm{H}$-phase electrode. The total cell size is $(30.0,0.718,15.0) \mathrm{nm}$. (b) The computational cell of the $2 \mathrm{D}$ transistors with $\mathrm{T}^{\prime}$-phase source and drain and a H-phase channel. The total cell size is $\left(10.0+L_{C}, 0.718,15.0\right) \mathrm{nm}$ with $L_{C}$ being 10.0, 18.0, or $20.0 \mathrm{~nm}$. The middle part shows the atomic configurations of the two investigated interface geometries seen from the top.

To simulate the electrostatic environment in a FET, both the 2D Schottky contacts and the transistors have been placed on top of a thin metal back gate and a dielectric region of $5 \mathrm{~nm}$ with a dielectric constant of 3.9 corresponding to $\mathrm{SiO}_{2}$. Both are described as a classical continuum. The lowest atom of the system is placed $2.4 \AA$ above the dielectric region. This value is chosen since it lies close to the experimental value of the distance between $2 \mathrm{H} \mathrm{MoS}_{2}$ and $\mathrm{SiO}_{2}$ [41] and since we have found that a variation between 2.4 and $4 \AA$ does not change the barrier height or depletion width. The electrostatic effect of the substrate is to screen the interface dipole, which increases the charge transfer since the potential difference between the metal and semiconductor is conserved despite the presence of the substrate. This increases the depletion width but conserves the barrier height.

We use a continuous doping model to add charge carriers to the semiconductor and we apply a p-type doping since $\mathrm{MoTe}_{2}$ most commonly exhibit a p-type intrinsic doping in ambient conditions $[8,42]$. The electrons per atom are explicitly modified by scaling the atomic densities [43]. We choose to dope all the atoms in the system to avoid any artificial effects of defining a sharp interface between the two phases. The extra holes in the metallic phase have a negligible effect.

\section{SCHOTTKY CONTACTS}

Our starting point is to investigate the two Schottky contacts. The results from these investigations will help to analyze the results of the simulations on the complete transistor setup. The p-doping level is varied between $10^{12}$ and $1.5 \times$ $10^{13} \mathrm{~cm}^{-2}$ and the effects on the Schottky barrier height, depletion width, and charge transport are investigated. The Schottky barrier height is found from the projected DOS as the distance from the Fermi level to the minima of the valence band. The valence-band edge is found by defining the gap region as energies at which the projected DOS is negligible. The evolution of the valence-band positions of the two contacts, when the doping is increased, is shown in Figs. 2(a) and 2(c). The Fermi level is placed at the energy zero point, and the $x$ coordinate of the leftmost Mo-atom, which belonged to the $\mathrm{H}$ phase before relaxation, is marked by a vertical gray line. The c-type contact only shows band bending for the three lowest doping levels. From then on, the valence bands are 

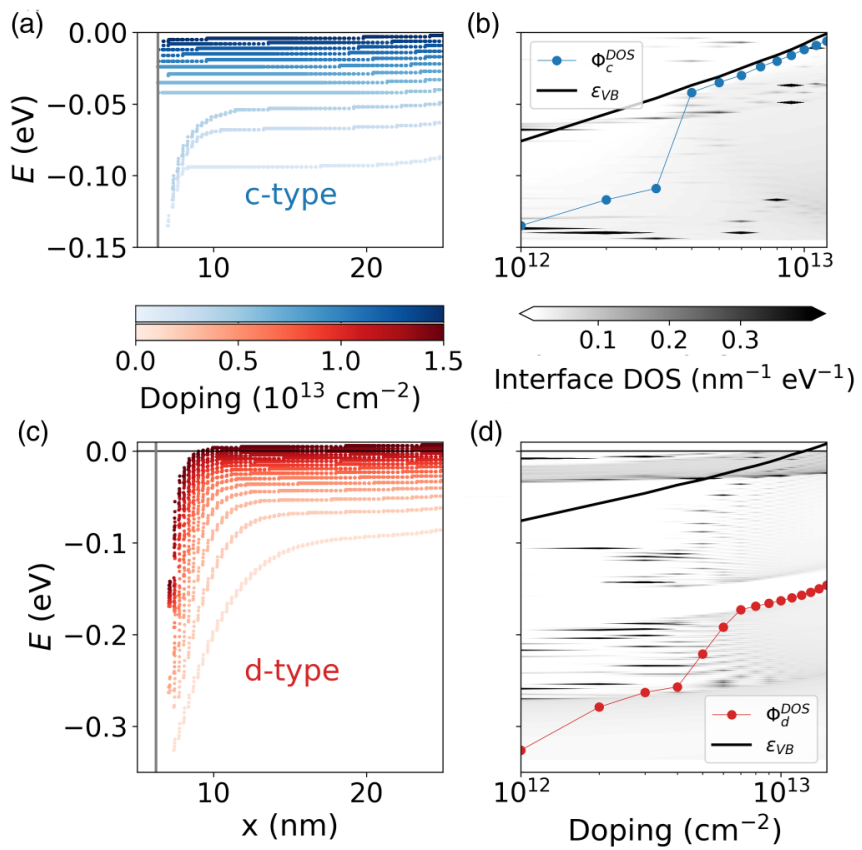

FIG. 2. The Schottky barrier evolution with varying levels of pdoping. (a and c) The valence-band edge of the $\mathrm{H}$ phase for the $\mathrm{c}$ - and d-type contacts, respectively. The gray line indicates the first Moatom belonging to the $\mathrm{H}$ phase. ( $\mathrm{b}$ and $\mathrm{d}$ ) The barrier heights as a function of the doping level (circles) along with the maximum of the valence band (black line). The DOS at the position of the gray line on (a) and (c) at each doping level is included as a gray-scale contour to illustrate the density of interface states between the Fermi level and the valence-band maximum.

flat and the barrier height coincides with the maximum of the valence band. The d-type contact show band bending for all the investigated doping levels.

The barrier height is shown together with the valenceband maxima as a function of the doping level in Figs. 2(b) and 2(d). The size of the barrier differs significantly between the two interface geometries and so does the behavior with the doping density. The barrier height does not vary smoothly with the doping level but rather jumps between energy plateaus. Our previous work [31] and the work by Liu et al. [25] have demonstrated that these heterophase $\mathrm{MoTe}_{2}$ contacts host interface states, which increases the tunneling probability. At the interfaces investigated here, some of these states bridge the Schottky barrier and result in both increased tunneling probability and an effectively lower barrier than what the strength of the interface dipole dictates. This effect is illustrated in Fig. 3. The interface dipole is created due to the depletion of holes in the valence band and the corresponding charge accumulation in the metal, which screens the depletion charge. The band bending, therefore, reflects the strength of the interface dipole and is an electrostatic effect. The DOS, on the other hand, is a representation of the available quantum states which electrons and holes can occupy. The barrier height extracted from the DOS, therefore, represents an effective barrier for quantum transport, which does not necessarily coincide with the electrostatic barrier. This effective barrier will in the following be referred to as the DOS

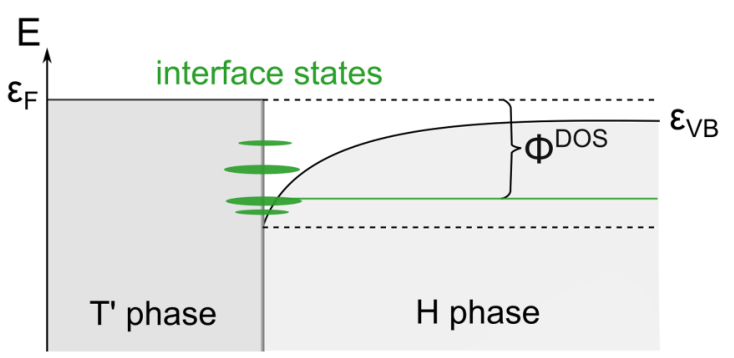

FIG. 3. The effect of interface states on the DOS barrier, $\Phi^{\mathrm{DOS}}$, between $\mathrm{H}$ - and $\mathrm{T}^{\prime}$-phase $\mathrm{MoTe}_{2}$. The electrostatic field from the interface dipole causes the valence band to bend downward creating a barrier height represented by the dashed black line. The DOS associated with interface states bridges this electrostatically defined barrier reducing the effective barrier for quantum transport illustrated by the green line.

barrier, $\Phi^{\mathrm{DOS}}$, in agreement with the definition in our previous work [31].

The density of interface states have been included in Figs. 2(b) and 2(d) as a contour plot. The contour represents the DOS at the position of the vertical gray line in Figs. 2(a) and 2(c). The states of interest lie at energies between the bottom of the barrier and the maximum of the valence band. Energies below this range host the valence-band states, and energies above cannot contribute to transport since these lie within the band gap of the H-phase electrode. The effect of the interface DOS is most easily seen in the case of the d-type contact. The barrier bottom is seen to jump between windows of low interface DOS around -0.17 and $-0.26 \mathrm{eV}$. Note that the slope of the interface states on this plot are much lower than the slope of the valence-band maxima. This demonstrates that the interface states originate from the $\mathrm{T}^{\prime}$ phase rather than the $\mathrm{H}$ phase, which was the case for the interface resonances in our previous study as well. The interface DOS illustrated in the contour plot can be associated with both regular metal-induced gap states, i.e., the exponential tail of bulk states in the metal, and such interface resonances.

These results illustrate that the barrier height depends nontrivially on the doping level due to the presence of interface states. We will now investigate how the interface dipole and depletion width vary with the doping level. In these ML contacts, the electronic density and the potential depend a lot on the atomic rearrangements at the phase boundary. To visualize how the interface dipole changes with the doping level, the electronic density of a contact with a low doping level, $N_{2 D}^{\text {ref }}=1 \times 10^{11} \mathrm{~cm}^{-2}$, is used as a reference. Almost no charge transfer is possible in the reference system since less than 0.02 holes are available in the entire semiconductor region. After the subtraction of the reference density, the doping difference between the reference device and this device is subtracted in order to remove the background doping density,

$$
\begin{aligned}
\Delta n_{e}(x)= & \frac{1}{W} \int\left[n_{e}(x, y, z)-n_{e}^{\mathrm{ref}}(x, y, z)\right] \mathrm{d} y \mathrm{~d} z \\
& -\left(N_{2 D}^{\mathrm{dop}}-N_{2 D}^{\mathrm{ref}}\right) .
\end{aligned}
$$

This difference in electronic density can be seen in Figs. 2(a) and 2(b) for the c-type and d-type contacts, 

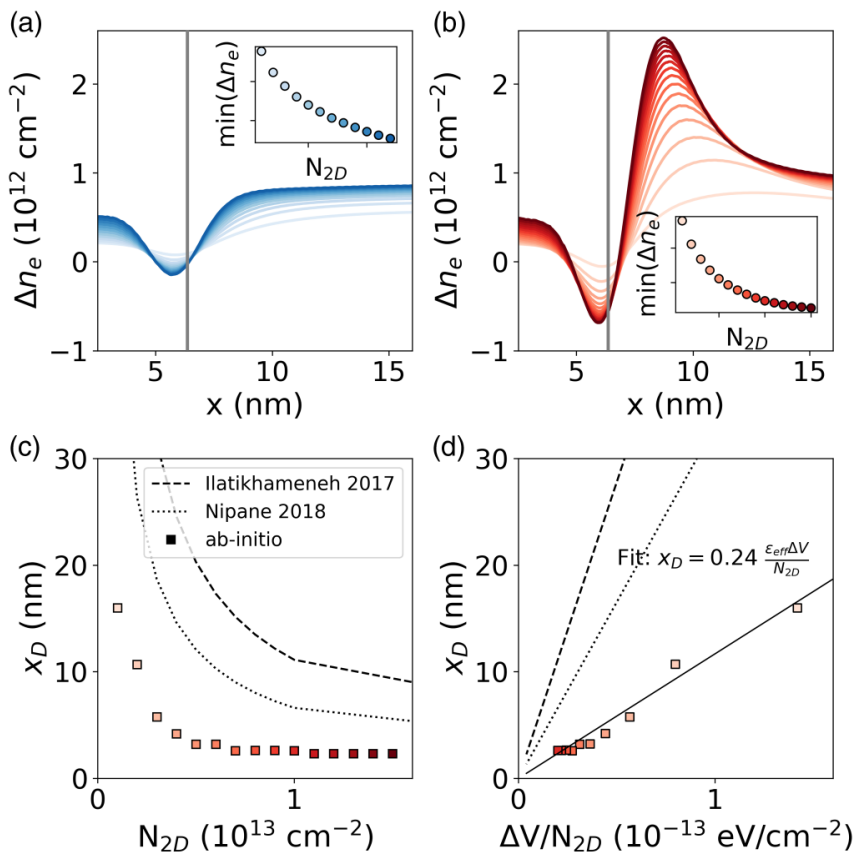

FIG. 4. The electron density at the two Schottky contacts (a and b) and the depletion width (c and d) of the d-type interface with varying p-doping following the color scale in Fig. 2. The depletion width is compared with the predictions by Ilatikhameneh et al. [44] (dashed line) and Nipane et al. [45] (dotted line). (d) The depletion width as a function of the work-function difference divided by the doping density and a linear fit to the calculated depletion widths (solid line).

respectively. The difference in the size of the dipole is quite clear. The c-type contact shows much less variation of the electronic density through the device, which is a footprint of a very small charge accumulation in the depletion region and correspondingly a small barrier height. This agrees with the trend in Fig. 2(a), where the barrier vanishes for doping levels above $3 \times 10^{13} \mathrm{~cm}^{-2}$. We expect that the decreased interface dipole at the c-type contact is related to a more favorably charge redistribution from the local atomic rearrangement at the interface compared with the d-type contact. The d-type contact shows a much larger variation and the interface dipole is seen to become more narrow in space as the doping is increased. The minimum of the density can be used to illustrate the smooth variation of the electronic density with the doping level and is shown in the insets.

Since the depletion width vanishes already at a doping level of $4 \times 10^{12} \mathrm{~cm}^{-2}$ in the c-type contact, we only consider how the depletion width varies with the doping level of the d-type contact. The depletion width can be found from the bending of the valence bands in Fig. 2(c). The values are compared in Figs. 4(c) and 4(d) to the expressions derived using classical electrostatics by Ilatikhameneh et al. [44] and Nipane et al. [45],

$$
\begin{aligned}
x_{D}^{\text {Ilatikhameneh }} & =\frac{\pi \varepsilon_{\mathrm{eff}} \Delta V}{\ln (4) q N_{2 D}}, \\
x_{D}^{\text {Nipane }} & =\frac{\pi^{2} \varepsilon_{\mathrm{eff}} \Delta V}{8 G q N_{2 D}} .
\end{aligned}
$$
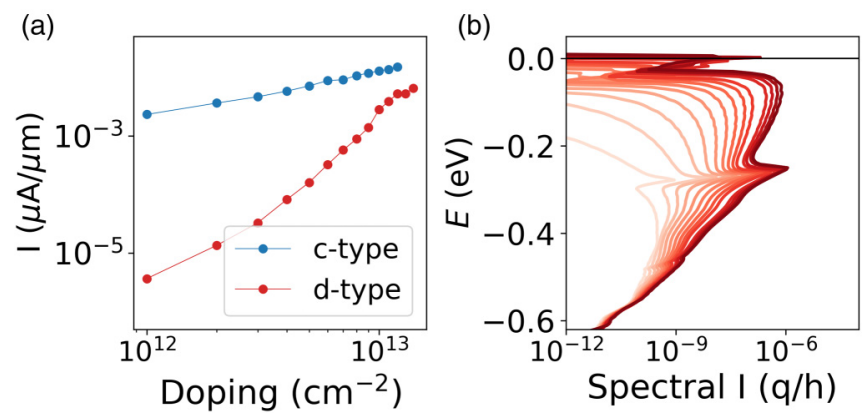

FIG. 5. Total and spectral current though the Schottky contacts at varying levels of p-doping. (a) The total current through the two contacts as a function of the doping level and at a bias of $V_{\text {bias }}=$ $10 \mathrm{mV}$. (b) shows the spectral current of the d-type contact at varying doping levels. The color scale follows Fig. 2.

$\Delta V$ is the work function difference between the two materials in the junction; $\varepsilon_{\text {eff }}$ is the effective dielectric constant which is determined by the dielectrics surrounding the $2 \mathrm{D}$ material, which in this case is $\varepsilon_{\text {eff }}=\left(\varepsilon_{\text {above }}+\varepsilon_{\text {below }}\right) / 2=$ $2.45 \varepsilon_{0} ; G \approx 0.915$ is Catalan's constant; and $q$ is the elementary charge. These depletion widths have been calculated using the work function of the $\mathrm{H}$ - and $\mathrm{T}^{\prime}$-phase electrodes at each doping level with computational parameters matching those used for the electrodes of the devices.

Comparing to the ab-initio results, it is clear that the depletion width cannot be predicted from the difference in work functions and doping level alone. First, the values of the two different phase boundaries, as already mentioned, differ a lot, and second, the depletion width of the d-type contact is considerably shorter than predicted by the classical models. However, as demonstrated by Fig. 4(d), the classically predicted linear scaling with $\Delta V / N_{2 D}$ seems to be fulfilled.

Finally, we investigate how the charge transport though the contact changes with the carrier density. We apply a small positive bias of $V_{\text {bias }}=10 \mathrm{mV}$ across the contacts. This drives a hole current from the metal to the semiconductor; the current can be calculated using the Landauer-Büttiker formula,

$$
\begin{aligned}
I= & \frac{2 q}{h} \int T\left(E, k_{\|}, \mu_{L}, \mu_{R}\right) \\
& \times\left[f\left(\frac{E-\mu_{L}}{k_{B} T}\right)-f\left(\frac{E-\mu_{R}}{k_{B} T}\right)\right] \mathrm{d} E .
\end{aligned}
$$

The $h$ is Plack's constant, $k_{B}$ is the Boltzmann constant, and $T\left(E, k_{\|}, \mu_{L}, \mu_{R}\right)$ is the energy- and k-dependent transmission coefficient, where $k_{\|}$is the k-vector parallel to the transport direction. The transmission is calculated from the retarded Green's function and the imaginary part of the electrode selfenergies as described in Ref. [39]. The $f$ is the Fermi-Dirac distribution; $\mu_{L}$ and $\mu_{R}$ are the chemical potentials of the two electrodes, defined as $\mu_{L}-\mu_{R}=q V_{\text {bias }}$; and $T$ is the temperature. We use $T=300 \mathrm{~K}$ to find the current at room temperature. The resulting currents through the two contacts are shown in Fig. 5(a). The current has a relatively smooth dependence on the carrier density and the magnitude differs about two orders of magnitude between the two geometries at low doping levels, but reaches a comparable value at high 
doping levels. Note that the current is shown on a logarithmic scale. The variation of the spectral current through the d-type contact is shown in Fig. 5(b). The spectral current is the term inside the integral in Eq. (2). Two peaks are present at the energies where a high and relatively constant interface DOS can be identified in Fig. 2(d) at all the investigated carrier densities. Those states, therefore, contribute significantly to the current regardless of the value of the Schottky barrier height, and we believe that they can be associated with interface resonances.

To summarize, the Schottky barrier in heterophase $\mathrm{MoTe}_{2}$ Schottky contacts can be electrostatically tuned to increase the current such that these can be utilized in a transistor. As expected, the interface dipole and charge transfer respond in a smooth manner when increasing the carrier density. This smooth increase is also reflected in the depletion width, which scales reciprocally with the carrier density. However, the size of the barrier height, the length of the depletion width, and the total magnitude of the current are determined by atomicscale and quantum effects. It has been established several times $[20,22,46]$, which we also confirm with these calculations, that the barrier height in the TMD heterojunctions depends on the local atomic positions at the phase boundary. From our investigation of the depletion width, we, therefore, naturally find that the magnitude of this quantity, as well as the size of the interface dipole, is highly dependent on the interface geometry as well. The barrier height behaves nontrivially when the carrier density is increased since interface states can bridge the barrier. Some of these interface states increase the transmission probability significantly and, therefore, also increase the current trough the contact.

\section{HETEROPHASE FETS}

After concluding these initial investigations of the isolated Schottky contacts, we are ready to simulate a complete transistor setup with source, drain, channel, and back gate as shown in Fig. 1(b). We combine the two Schottky contacts to create transistors with channel lengths of 10,18 , and $20 \mathrm{~nm}$ and choose a doping level of $N_{A}=5 \times 10^{12} \mathrm{~cm}^{-2}$.

The transfer characteristics of the 2D FETs are shown in Fig. 6 at a bias of $V_{\text {bias }}=-10 \mathrm{mV}$. This bias results in a device with a d-type barrier at the source and flat bands at the drain. The transfer characteristics are calculated by gradually varying the potential between the back gate and the source electrode and calculating the current using Eq. (2). At a gate potential of $3.0 \mathrm{~V}$ in the case of the $10 \mathrm{~nm}$ channel and $2.4 \mathrm{~V}$ in the case of the other two devices, the current is switched completely off. Above those potentials, the devices shift polarity and begin to be dominated by electron transport in the conduction band. The subthreshold regimes where the current rises exponentially are indicated as the shaded red regions in Fig. 6. The subthreshold swings (SS) in this region are $0.24,0.14$, and $0.12 \mathrm{~V} / \mathrm{dec}$ for the 10,18 , and $20 \mathrm{~nm}$ FETs, respectively. The values of the 18 - and 20 -nm devices agree reasonably well with the SS value of a heterophase $\mathrm{MoS}_{2}$ device fabricated by Nourbakhsh et al. [21] of $120 \mathrm{mV} / \mathrm{dec}$, whereas it is considerably larger than the SS value measured in a $\mathrm{MoTe}_{2}$ heterophase device fabricated by Zhang et al.

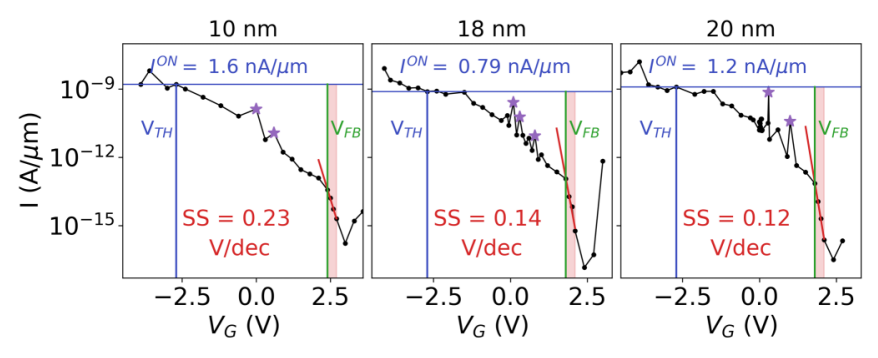

FIG. 6. Transfer characteristics of the heterophase FETs at $V_{\text {bias }}=-10 \mathrm{mV}$. The ON-currents and SS are indicated. The SS values are found from the fit indicated by the red lines and the subthreshold regimes are indicated by the shaded red regions. The flat-band condition, $V_{F B}$, is marked by the green lines and the threshold potential at $V_{T H}=-2.7 \mathrm{~V}$ by the blue lines. Purple stars indicate rises in the current due to resonant tunneling.

[11] of merely $69 \mathrm{mV} / \mathrm{dec}$. The impressively low SS value in the latter device is most likely due to the optimized setup used in this case. The heterophase transistor was placed on the high- $\kappa$ dielectric $\mathrm{HfO}_{2}$ with an equivalent oxide thickness (EOT) of $1.87 \mathrm{~nm}$ and the back gate positioned only below the $2 \mathrm{H}$ phase. A low EOT generally both decreases the SS value and increases the ON-current in a device [47].

In the Supplemental Material Ref. [35] we use a classical electrostatic model to estimate how an increment in the applied gate potential will result in a potential increment at the position of the semiconductor in a device setup such as ours. The relation between the two is given by the ideality factor, $\eta$, which determines the SS value, $\mathrm{SS}=\eta \times 60$ $\mathrm{mV} / \mathrm{dec}$. The model assumes a constant charge density in the channel and estimates the effect of the geometry of the device, which creates a capacitive potential divider formed by the gate capacitance in series with the capacitance of the substrate and vacuum region above the transistor. This model predicts SS values of $0.20,0.12$, and $0.12 \mathrm{~V} / \mathrm{dec}$ for the 10,18 , and $20 \mathrm{~nm}$ channels, respectively. Most of the deviation from the thermodynamic limit of $\mathrm{SS}=60 \mathrm{mV} / \mathrm{dec}$ can, therefore, be explained by the setup of the device. The remaining discrepancy is most likely due to the variation in the charge density along the channel and the tunneling between the source and the drain. The source-drain tunneling is most important in the 10-nm device, which explains why the SS value of this device is very large.

The flat-band condition, $V_{F B}$, is met when the exponential dependence of the current breaks off. This point is marked by the vertical green line in Fig. 6. At lower gate potentials, the devices enter the Schottky barrier regime where the current is conducted as a mixture of thermionic emission and tunneling of the holes. The threshold potential at $V_{G}=V_{T H}=-2.7 \mathrm{~V}$ is defined as the point where the valence-band edge rises above the chemical potential of the source and has been identified by calculating the projected DOS. In this condition, we find ONcurrents of 1.6, 0.79 , and $1.2 \mathrm{nA} / \mu \mathrm{m}$, respectively. Previous experimental measurements on $\mathrm{MoTe}_{2} 2 \mathrm{H}-1 \mathrm{~T}^{\prime}$ heterophase devices with channel thickness between 5 and $8 \mathrm{~nm}$ and channel lengths on the $\mu \mathrm{m}$ scale have presented ON-currents around $15 \mathrm{nA} / \mu \mathrm{m}$ at a bias of $50 \mathrm{mV}$ and $39.6 \mathrm{nA} / \mu \mathrm{m}$ at a bias of $0.1 \mathrm{~V}[8,9]$. By non-self-consistently increasing the 
TABLE I. EOT, $t_{E O T}$; channel thicknesses, $t_{c h}$; channel lengths, $L_{C}$; ON-currents, $I^{O N}$; power supply voltages, $V_{D D}$; and SS of MoTe ${ }_{2}$-based heterophase devices compared with the IRDS 2022 goals [1]. *Extrapolated non-self-consistently.

\begin{tabular}{|c|c|c|c|c|c|c|c|}
\hline Ref. & $t_{E O T}$ & $t_{c h}$ & $L_{C}$ & $I^{O N}$ & $V_{s d}\left(I^{O N}\right)$ & $V_{D D}$ & SS \\
\hline Zhang et al. [11] & $1.87 \mathrm{~nm}$ & $8 \mathrm{~nm}$ & $4 \mu \mathrm{m}$ & $0.25 \mu \mathrm{A} / \mu \mathrm{m}$ & $0.1 \mathrm{~V}$ & $0.8 \mathrm{~V}$ & $69 \mathrm{mV} / \mathrm{dec}$ \\
\hline Sung et al. [8] & $300 \mathrm{~nm}$ & $5-6 \mathrm{~nm}$ & $4-15 \mu \mathrm{m}$ & $15 \mathrm{nA} / \mu \mathrm{m}$ & $50 \mathrm{mV}$ & $40 \mathrm{~V}$ & \\
\hline Ma et al. [9] & $300 \mathrm{~nm}$ & $8 \mathrm{~nm}$ & $20 \mu \mathrm{m}$ & $39.6 \mathrm{nA} / \mu \mathrm{m}$ & $-0.1 \mathrm{~V}$ & $100 \mathrm{~V}$ & \\
\hline This work & $5 \mathrm{~nm}$ & ML & $10 \mathrm{~nm}$ & $1.6 \mathrm{nA} / \mu \mathrm{m}$ & $-10 \mathrm{mV}$ & $5.7 \mathrm{~V}$ & $0.23 \mathrm{~V} / \mathrm{dec}$ \\
\hline This work & $5 \mathrm{~nm}$ & ML & $18 \mathrm{~nm}$ & $0.79 \mathrm{nA} / \mu \mathrm{m}$ & $-10 \mathrm{mV}$ & $5.1 \mathrm{~V}$ & $0.14 \mathrm{~V} / \mathrm{dec}$ \\
\hline This work & $5 \mathrm{~nm}$ & ML & $20 \mathrm{~nm}$ & $1.2 \mathrm{nA} / \mu \mathrm{m}$ & $-10 \mathrm{mV}$ & $5.1 \mathrm{~V}$ & $0.12 \mathrm{~V} / \mathrm{dec}$ \\
\hline This work* & $5 \mathrm{~nm}$ & ML & $20 \mathrm{~nm}$ & $21 \mathrm{nA} / \mu \mathrm{m}$ & $-0.1 \mathrm{~V}$ & - & - \\
\hline This work* & $5 \mathrm{~nm}$ & ML & $20 \mathrm{~nm}$ & $0.15 \mathrm{~mA} / \mu \mathrm{m}$ & $-0.7 \mathrm{~V}$ & - & - \\
\hline IDRS 2022[1] & $1 \mathrm{~nm}$ & $6 \mathrm{~nm}^{\mathrm{a}}$ & $16 \mathrm{~nm}$ & $912 \mu \mathrm{A} / \mu \mathrm{m}$ & $0.7 \mathrm{~V}$ & $0.7 \mathrm{~V}$ & $82 \mathrm{mV} / \mathrm{dec}$ \\
\hline
\end{tabular}

${ }^{a}$ Fin width of the finFET design.

bias, we can compare our ON-currents with these values and get a reasonable agreement. The extrapolated $\mathrm{ON}$-currents of the 20-nm device are summarized in Table I, and the extrapolated ON-currents of the two other devices can be found in the Supplemental Material Ref. [35]. In the table, we also include the measurements on the optimized device fabricated by Zhang et al. [11], which results in an ON-current that is two orders of magnitude higher than the other measurements and those of our results.

The energy efficiency of the devices is reflected both in the value of the SS and in the power supply voltage, $V_{D D}$. We define $V_{D D}$ as the gate potential difference between the threshold potential and the potential of minimal current. This value is related to the Schottky barrier height since a lower barrier at the source will result in a lower flat-band potential and decrease this value. The $10-\mathrm{nm}$ device has a $V_{D D}$ value of $5.7 \mathrm{~V}$ and the 18 - and 20-nm devices have $V_{D D}$ values of $5.1 \mathrm{~V}$. These values are around an order of magnitude higher than what typical silicon-based devices require [1]. Note that due to the relatively large barrier at the d-contact, it is the Schottky barrier regime rather than the subthreshold regime that dominates the size of $V_{D D}$ and the overall device efficiency. The power supply voltage required in the $10-\mathrm{nm}$ device is larger than in the two other devices due to the proximity of the contacts. The interface dipole fields at the source and drain interacts, which results in a less efficient gate response and a larger flat-band potential. The effect is illustrated in the Supplemental Material Ref. [35]. In the 10-nm device, the OFF-current is also larger than in the other devices since this device isn't fully turned off when the switch in polarity occurs. It is important to note that the switch in polarity is directly related to the band gap of the channel. PBE systematically underestimates the band gap of the 2D TMDs, which means that the potential of the polarity switch is underestimated as well.

The Schottky barrier regime between $V_{T H}$ and $V_{F B}$ is dominated by large spikes in the current, which are marked by purple stars in Fig. 6. The spikes in the current occur due to resonant tunneling and the effect is especially pronounced in the 20-nm device. In Fig. 7, the spectral current of this device is shown at different gate potentials between the first purple star at $V_{G}=V_{Q R 1}=1.0 \mathrm{~V}$ and the threshold potential. As the device is turned on, the peaks associated with the quantized states in the channel appear and disappear whenever a quantized state contributes to the tunneling current. At the two potentials corresponding to the purples stars, large peaks due to resonant tunneling can be identified at low energies.

The effect is most pronounced at a gate potential of $0.3 \mathrm{~V}$. In Figs. 8(a) and 8(b), the projected DOS at this gate potential is shown along with the transmission spectrum. Two kinds of quantum phenomena cause the peaks to occur in the transmission and spectral current. The first type is the interface states discussed in the previous chapter, which have been highlighted in green in the figure; the second type are standing waves in the channel that form due to quantum confinement. These have been marked in blue. In these devices, extraordinary large peaks occur in the transmission when a standing wave bridges the Schottky barrier. An example of this is highlighted by the blue arrows in the figure.

Note that the transmission is quite low even below the bottom of the barrier. This is due to the restriction of momentum conservation in ballistic transport. In p-type $\mathrm{MoTe}_{2}$ heterophase contacts, the ballistic transport is significantly reduced since only very few k-points along the $Y \rightarrow \Gamma$ path are occupied both in the metallic and in the semiconducting phase. Conserving the momentum perpendicular to the transport direction is, therefore, only possible for a narrow range of k-points. This is illustrated in Fig. 8(c), where the $k_{y}$ dependence of the transmission is illustrated. We, therefore, expect that including the effects of electron phonon scattering might actually increase the device performance.

To summarize, we find that resonant tunneling and momentum conservation play a very large role for the device performance even in these relatively long channel devices. Comparing the behavior of the Schottky contacts, we find that the standing waves in the channel affect the current to a larger degree than the effect of the interface resonances. Since the standing waves are a property of the semiconductor and not the metal, the electrostatic field from the gate moves these significantly up and down in energy. When a standing wave bridges the Schottky barrier, the current increases dramatically. This results in large current fluctuations in the transfer characteristics with peaks in the current rising more than an order of magnitude. As it demonstrated in Figs. 8(a) and 8 (b), the interface states also contribute to the current in the transistors, but the effect is smaller and relatively constant with the gate potential since they have origin in the metallic phase. 

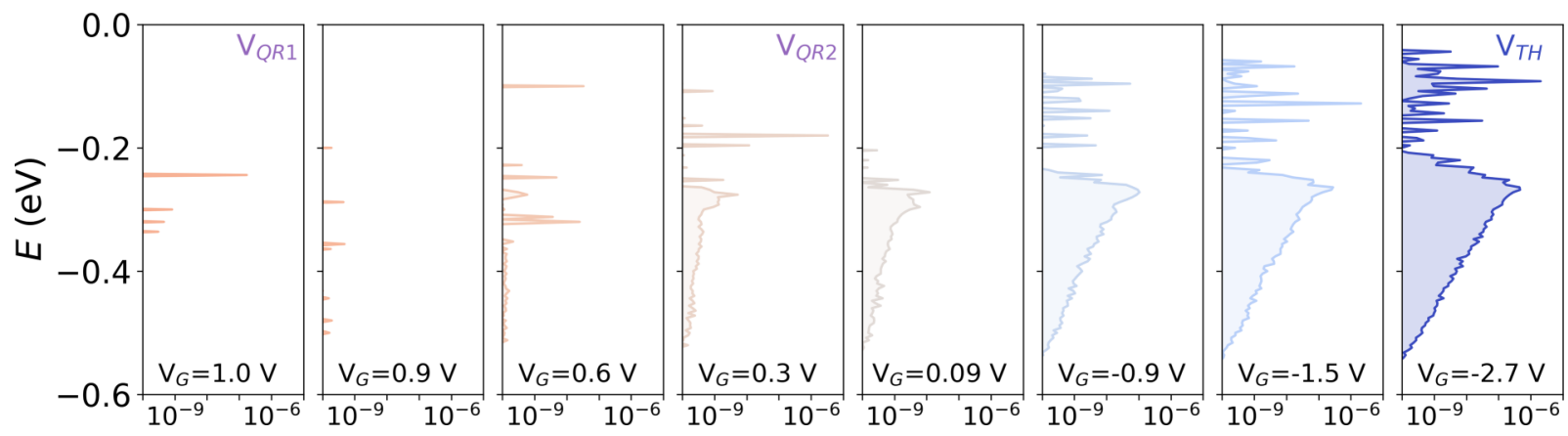

FIG. 7. Spectral current in units of $\mathrm{q} / \mathrm{h}$ in the transistor with a channel length of $20 \mathrm{~nm}$. The two large current peaks marked by the purple stars on Fig. 6 are denoted QR1 and QR2. At the quantum resonances (QR), significant contributions in the tunneling current occur through these peaks.

\section{DISCUSSION}

Previous calculations on similar devices have been performed by Fan et al. [30]. These devices differ from ours since the $\mathrm{T}$ phase rather than $\mathrm{T}^{\prime}$ phase was used as contacts, the channel lengths were much smaller, between 5 and $8 \mathrm{~nm}$, the interface was along the armchair direction, and the devices had both a top and a back gate with EOTs of only $0.5 \mathrm{~nm}$. Furthermore, that study used the local density approximation functional rather than the PBE functional. The results from those calculations showed ON-currents which are around an order of magnitude larger than those reported here and a power supply voltage around $1 \mathrm{~V}$. For $\mathrm{WTe}_{2}$ - and $\mathrm{WSe}_{2}$-based devices, the results from Fan et al. suggest that devices based on the $\mathrm{T}$ phase generally conduct more current than $\mathrm{T}^{\prime}$-based devices. The reason for this difference might lie in the difference in dispersion between the $\mathrm{T}$ and the $\mathrm{T}^{\prime}$ phases. Since the $\mathrm{T}$ phase is metallic, whereas the $\mathrm{T}^{\prime}$ phase is semimetallic, the states are generally more densely distributed in k-space, which allows for a greater probability of ballistic transport of carriers. Whether the rest of the difference in the ON-current results from the difference in the device setup or in the method between this study and ours is difficult to say. However, the behavior of the interfaces along the zigzag direction seems

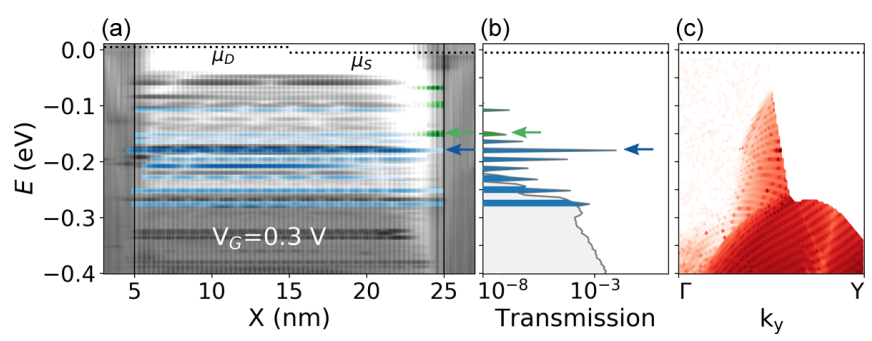

FIG. 8. Quantum effects in $\mathrm{MoTe}_{2}$ heterophase transistors. (a) shows the projected DOS of the $20 \mathrm{~nm}$ transistor at a gate potential of $V_{G}=0.3 \mathrm{~V}$. The chemical potential of the drain, $\mu_{D}$, and the source $\mu_{S}$ are indicated by the dotted black lines. The interface states are marked by green contours and some of the standing waves in blue contours. (b) The transmission spectrum where the peaks due to the standing waves and interface states have been marked in blue and green. (c) The k-resolved transmission perpendicular to the transport direction. The most predominant peak in the transmission spectrum is marked by the blue arrows. most relevant since the experimental evidence suggests that the interfaces occur along this direction $[8,33,34]$.

In Table I, we have summarized our results and compared it with the three experimental studies mentioned in the previous section and with the International Roadmap for Devices and Systems (IRDS) requirements [1] of a 2022 high-performance logic device. In summary, our 18- and 20-nm devices show a performance, which is comparable to previous measurements on devices on top of $\mathrm{SiO}_{2}$ substrates. Compared with the IRDS goal, the energy efficiency and total ON-current of the devices are not competitive with the performance of silicon-based devices. The experimental results by Zhang et al. [11], on the other hand, suggest that the IRDS requirements might be fulfilled by using a high- $\kappa$ dielectric as the gate oxide, which is also the common practice in silicon-based devices. However, the device dimensions in the study by Zhang et al. are much larger than both our calculations and the IRDS requirements. This, therefore, opens the question of whether the heterophase design will be competitive when scaling down the channel length of the devices. Judging from these considerations, the idealized ML limit with perfect interfaces might not be preferable since ballistic transport through such geometries is limited by momentum conservation. However, electron-phonon scattering might remove some of this effect and since the device performance is dominated by the local atomic configuration at the interface, the presence of defects in the interface might also dramatically alter the behavior. Furthermore, as demonstrated for the Schottky contacts, a high carrier density will smear out the difference between interface geometries and result in a relatively low Schottky barrier. A heavy doping of the $\mathrm{H}$ phase might, therefore, be a viable approach to obtain a competitive design.

\section{CONCLUSION}

Our simulations of the heterophase FET devices demonstrated that quantum effects play a large role in the performance of these devices even at a channel length of $20 \mathrm{~nm}$. We find that QRs result in resonant tunneling and large fluctuations in the current in the Schottky barrier regime of the transfer characteristics. The QRs stem from both interface states at the contacts and standing waves in the channel. The interface states are a material-specific property of the devices, whereas the standing waves are an effect of the 
scale and dimensionality of the device and can be expected to be a general property of $2 \mathrm{D}$ transistors. Furthermore, we find that the restriction of momentum conservation inhibits the ballistic transport significantly in these idealized devices.

In conclusion, our results demonstrate many challenges related to the heterophase FET design. Effects from electronphonon scattering and interface defects might actually improve the performance, and calculations estimating such effects could shed light on this possibility. Likewise, moving from the ML to the few-layer limit or ensuring a high carrier density in the channel might resolve some of the difficulties since this will somewhat smear out some of the dependency on the interface geometry. The results by Nourbakhsh et al. [21], where $\mathrm{MoS}_{2}$-based heterophase devices with both one and three layers were investigated, showed that the three-layer device performs better, which agrees with this conclusion.

\section{ACKNOWLEDGMENTS}

This work is partly funded by the Innovation Fund Denmark (IFD) under File No. 5189-00082B. We thank Kurt Stokbro and Daniele Stradi for their contributions.
[1] IEEE International Roadmap for Devices and Systems (2020).

[2] D. Akinwande, C. Huyghebaert, C.-H. Wang, M. I. Serna, S. Goossens, L.-J. Li, H.-S. P. Wong, and F. H. L. Koppens, Graphene and two-dimensional materials for silicon technology, Nature (London) 573, 507 (2019).

[3] B. Balasubramaniam, N. Singh, P. Kar, A. Tyagi, J. Prakash, and R. K. Gupta, Engineering of transition metal dichalcogenide based 2D nanomaterials through doping for environmental applications, Mol. Syst. Des. Eng. 4, 804 (2019).

[4] A. Allain, J. Kang, K. Banerjee, and A. Kis, Electrical contacts to two-dimensional semiconductors, Nat. Mater. 14, 1195 (2015).

[5] C. Kim, I. Moon, D. Lee, M. S. Choi, F. Ahmed, S. Nam, Y. Cho, H. J. Shin, S. Park, and W. J. Yoo, Fermi level pinning at electrical metal contacts of monolayer molybdenum dichalcogenides, ACS Nano 11, 1588 (2017).

[6] K.-A. N. Duerloo, Y. Li, and E. J. Reed, Structural phase transitions in two-dimensional Mo- and W-dichalcogenide monolayers, Nat. Commun. 5, 4214 (2014).

[7] Y. Yoo, Z. P. DeGregorio, Y. Su, S. J. Koester, and J. E. Johns, In-plane $2 \mathrm{H}-1 \mathrm{~T}{ }^{\prime} \mathrm{MoTe}_{2}$ homojunctions synthesized by fluxcontrolled phase engineering, Adv. Mater. 29, 1605461 (2017).

[8] J. H. Sung, H. Heo, S. Si, Y. H. Kim, H. R. Noh, K. Song, J. Kim, C.-S. Lee, S.-Y. Seo, D.-H. Kim, H. K. Kim, H. W. Yeom, T.-H. Kim, S.-Y. Choi, J. S. Kim, and M.-H. Jo, Coplanar semiconductor-metal circuitry defined on few-layer $\mathrm{MoTe}_{2}$ via polymorphic heteroepitaxy, Nat. Nanotechnol. 12, 1064 (2017).

[9] R. Ma, H. Zhang, Y. Yoo, Z. P. Degregorio, L. Jin, P. Golani, J. Ghasemi Azadani, T. Low, J. E. Johns, L. A. Bendersky, A. V. Davydov, and S. J. Koester, $\mathrm{MoTe}_{2}$ Lateral homojunction field-effect transistors fabricated using flux-controlled phase engineering, ACS Nano 13, 8035 (2019).

[10] X. Zhang, Z. Jin, L. Wang, J. A. Hachtel, E. Villarreal, Z. Wang, T. Ha, Y. Nakanishi, C. S. Tiwary, J. Lai, L. Dong, J. Yang, R. Vajtai, E. Ringe, J. C. Idrobo, B. I. Yakobson, J. Lou, V. Gambin, R. Koltun, and P. M. Ajayan, Low Contact Barrier in $2 \mathrm{H} / 1 \mathrm{~T}^{\prime} \mathrm{MoTe}_{2}$ In-plane heterostructure synthesized by chemical vapor deposition, ACS Appl. Mater. Interfaces 11, 12777 (2019).

[11] Q. Zhang, X.-F. Wang, S.-H. Shen, Q. Lu, X. Liu, H. Li, J. Zheng, C.-P. Yu, X. Zhong, L. Gu, T.-L. Ren, and L. Jiao, Simultaneous synthesis and integration of two-dimensional electronic components, Nat. Electron. 2, 164 (2019).

[12] X. Xu, S. Chen, S. Liu, X. Cheng, W. Xu, P. Li, Y. Wan, S. Yang, W. Gong, K. Yuan, P. Gao, Y. Ye, and L. Dai,
Millimeter-scale single-crystalline semiconducting $\mathrm{MoTe}_{2}$ via solid-to-solid phase transformation, J. Am. Chem. Soc. 141, 2128 (2019).

[13] X. Xu, S. Liu, B. Han, Y. Han, K. Yuan, W. Xu, X. Yao, P. Li, S. Yang, W. Gong, D. A. Muller, P. Gao, Y. Ye, and L. Dai, Scaling-up atomically thin coplanar semiconductor-metal circuitry via phase engineered chemical assembly, Nano Lett. 19, 6845 (2019).

[14] S. Cho, S. Kim, J. H. Kim, J. Zhao, J. Seok, D. H. Keum, J. Baik, D.-H. Choe, K. J. Chang, K. Suenaga, S. W. Kim, Y. H. Lee, and $\mathrm{H}$. Yang, Phase patterning for ohmic homojunction contact in $\mathrm{MoTe}_{2}$, Science 349, 625 (2015).

[15] S. Song, D. H. Keum, S. Cho, D. Perello, Y. Kim, and Y. H. Lee, Room Temperature Semiconductor-Metal Transition of $\mathrm{MoTe}_{2}$ Thin films engineered by strain, Nano Lett. 16, 188 (2016).

[16] Y. Katagiri, T. Nakamura, A. Ishii, C. Ohata, M. Hasegawa, S. Katsumoto, T. Cusati, A. Fortunelli, G. Iannaccone, G. Fiori, S. Roche, and J. Haruyama, Gate-tunable atomically thin lateral $\mathrm{MoS}_{2}$ Schottky junction patterned by electron beam, Nano Lett. 16, 3788 (2016).

[17] W. S. Paz and J. J. Palacios, A theoretical study of the electrical contact between metallic and semiconducting phases in monolayer $\mathrm{MoS}_{2}$, 2D Materials 4, 015014 (2016).

[18] M. Houssa, R. Meng, V. Afanas'ev, and A. Stesmans, Firstprinciples study of the contact resistance at 2D Metal/2D semiconductor heterojunctions, Appl. Sci. 10, 2731 (2020).

[19] D. Saha and S. Mahapatra, Atomistic modeling of the metallicto-semiconducting phase boundaries in monolayer $\mathrm{MoS}_{2}$, Appl. Phys. Lett. 108, 253106 (2016).

[20] D. Saha and S. Mahapatra, Asymmetric junctions in metallicsemiconducting-metallic heterophase $\mathrm{MoS}_{2}$, IEEE Trans. Electron Devices 64, 2457 (2017).

[21] A. Nourbakhsh, A. Zubair, R. N. Sajjad, T. K. Amir, W. Chen, S. Fang, X. Ling, J. Kong, M. S. Dresselhaus, E. Kaxiras, K. K. Berggren, D. Antoniadis, and T. Palacios, $\mathrm{MoS}_{2}$ Field-effect transistor with sub-10 nm channel length, Nano Lett. 16, 7798 (2016).

[22] M. L. Urquiza and X. Cartoixà, Schottky barriers, emission regimes and contact resistances in $2 \mathrm{H}-1 \mathrm{~T}^{\prime} \mathrm{MoS}_{2}$ lateral metalsemiconductor junctions from first-principles, 2D Materials 7, 045030 (2020).

[23] X.-W. Jiang, L.-W. Wang, Z.-Q. Fan, J.-W. Luo, S.-S. Li, L.Y. Jiao, and R. Huang, In-plane Schottky-barrier field-effect transistors based on $1 \mathrm{~T} / 2 \mathrm{H}$ heterojunctions of transition-metal dichalcogenides, Phys. Rev. B 96, 165402 (2017). 
[24] H. F. Bai, L. C. Xu, M. Y. Di, L. Y. Hao, Z. Yang, R. P. Liu, and $\mathrm{X}$. Y. Li, The intrinsic interface properties of the top and edge 1T/2H MoS 2 contact: A first-principles study, J. Appl. Phys. 123, 095301 (2018).

[25] S. Liu, J. Li, B. Shi, X. Zhang, Y. Pan, M. Ye, R. Quhe, Y. Wang, H. Zhang, J. Yan, L. Xu, Y. Guo, F. Pan, and J. Lu, Gate-tunable interfacial properties of in-plane ML MX $\mathrm{MX}_{2} 1 \mathrm{~T}^{\prime}-2 \mathrm{H}$ heterojunctions, J. Mater. Chem. C 6, 5651 (2018).

[26] E. Yang, J. E. Seo, D. Seo, and J. Chang, Intrinsic limit of contact resistance in the lateral heterostructure of metallic and semiconducting $\mathrm{PtSe}_{2}$, Nanoscale 12, 14636 (2020).

[27] D. Marian, E. Dib, T. Cusati, E. G. Marin, A. Fortunelli, G. Iannaccone, and G. Fiori, Transistor Concepts Based on Lateral Heterostructures of Metallic and Semiconducting Phases of $\mathrm{MoS}_{2}$, Phys. Rev. Appl. 8, 054047 (2017).

[28] Z. Dong and J. Guo, On low-resistance contacts to 2-D MoTe by crystalline phase junctions, IEEE Trans. Electron Devices 65, 1583 (2018).

[29] A. Li, J. Pan, X. Dai, and F. Ouyang, Electrical contacts of coplanar 2H/1T' MoTe $_{2}$ monolayer, J. Appl. Phys. 125, 075104 (2019).

[30] Z. Q. Fan, X. W. Jiang, J. Chen, and J. W. Luo, Improving performances of in-plane transition-metal dichalcogenideSchottky barrier field-effect transistors, ACS Appl. Mater. Interfaces 10, 19271 (2018)

[31] L. Jelver, D. Stradi, K. Stokbro, and K. W. Jacobsen, Schottky barrier lowering due to interface states in $2 \mathrm{D}$ heterophase devices, Nanoscale Adv. 3, 567 (2021).

[32] Á. Szabó, C. Klinkert, D. Campi, C. Stieger, N. Marzari, and M. Luisier, Ab initio simulation of band-to-band tunneling fets with single- and few-layer 2-d materials as channels, IEEE Trans. Electron Devices 65, 4180 (2018).

[33] X. Xu, B. Han, S. Liu, S. Yang, X. Jia, W. Xu, P. Gao, Y. Ye, and L. Dai, Atomic-Precision Repair of a Few-Layer 2H-MoTe Thin film by phase transition and recrystallization induced by a heterophase interface, Adv. Mater. 32, 2000236 (2020).

[34] B. Han, C. Yang, X. Xu, Y. Li, R. Shi, K. Liu, H. Wang, Y. Ye, J. Lu, D. Yu, and P. Gao, Correlating the electronic structures of metallic/semiconducting $\mathrm{MoTe}_{2}$ interface to its atomic structures, Natl. Sci. Rev. 8, nwaa087 (2020).

[35] See Supplemental Material at http://link.aps.org/supplemental/ 10.1103/PhysRevB.104.045313 for for a stability analysis of different interface geometries, extrapolated values of the oncurrent at a larger bias, the model for estimating the sub-threshold slope, and illustrations of the dipole interaction.

[36] P. Hohenberg and W. Kohn, Inhomogeneous electron gas, Phy. Rev. 136, B864 (1964).

[37] W. Kohn and L. J. Sham, Self-consistent equations including exchange and correlation effects, Phys. Rev. 140, A1133 (1965).

[38] J. P. Perdew, K. Burke, and M. Ernzerhof, Generalized Gradient Approximation Made Simple, Phys. Rev. Lett. 77, 3865 (1996).

[39] S. Smidstrup, T. Markussen, P. Vancraeyveld, J. Wellendorff, J. Schneider, T. Gunst, B. Verstichel, D. Stradi, P. A. Khomyakov, U. G. Vej-Hansen, M.-E. Lee, S. T. Chill, F. Rasmussen, G. Penazzi, F. Corsetti, A. Ojanperä, K. Jensen, M. L. N. Palsgaard, U. Martinez, A. Blom et al., QuantumATK: An integrated platform of electronic and atomic-scale modelling tools, J. Phys. Condens. Matter 32, 015901 (2019).

[40] M. van Setten, M. Giantomassi, E. Bousquet, M. Verstraete, D. Hamann, X. Gonze, and G.-M. Rignanese, The PseudoDojo: Training and grading a 85 element optimized norm-conserving pseudopotential table, Comput. Phys. Commun. 226, 39 (2018).

[41] J. A. Woollam and R. B. Somoano, Superconducting critical fields of alkali and alkaline-earth intercalates of $\operatorname{mos}_{2}$, Phys. Rev. B 13, 3843 (1976).

[42] J. Liu, Y. Wang, X. Xiao, K. Zhang, N. Guo, Y. Jia, S. Zhou, Y. Wu, Q. Li, and L. Xiao, Conversion of multi-layered MoTe2 transistor between P-type and N-type and their use in inverter, Nanoscale Res. Lett. 13, 291 (2018).

[43] The details on the doping methods implemented in the atk code can be found in the quantumatk technical notes (2021).

[44] H. Ilatikhameneh, T. Ameen, F. Chen, H. Sahasrabudhe, G. Klimeck, and R. Rahman, Dramatic impact of dimensionality on the electrostatics of PN junctions, arXiv:1704.05488 (2017).

[45] A. Nipane, S. Jayanti, A. Borah, and J. T. Teherani, Electrostatics of lateral p-n junctions in atomically thin materials, J. Appl. Phys. 122, 194501 (2017).

[46] P. Li, Y. Wen, X. He, Q. Zhang, C. Xia, Z.-M. Yu, S. A. Yang, Z. Zhu, H. N. Alshareef, and X.-X. Zhang, Evidence for topological type-II Weyl semimetal eWTe2, Nat. Commun. 8, 2150 (2017).

[47] M. Houssa, D. Athanasios, and A. Molle, 2D Materials for Nanoelectronics (CRC Press, Boca Raton, 2016). 